\title{
AUSLANDER'S $\delta$-INVARIANTS OF GORENSTEIN LOCAL RINGS
}

\author{
SONGQING DING
}

(Communicated by Eric Friedlander)

\begin{abstract}
Let $(R, \mathfrak{m}, k)$ be a Gorenstein local ring with associated graded ring $G(R)$. It is conjectured that for any integer $n>0$, Auslander's $\delta$ invariant $\delta\left(R / \mathfrak{m}^{n}\right)$ of $R / \mathfrak{m}^{n}$ equals 1 if and only if $\mathfrak{m}^{n}$ is contained in a parameter ideal of $R$. In an earlier paper we showed that the conjecture holds if $G(R)$ is Cohen-Macaulay. In this paper we prove that the conjecture has an affirmative answer if $\operatorname{depth} G(R)=\operatorname{dim} R-1$ and $R$ is gradable. We also prove that if $R$ is not regular and $\operatorname{depth} G(R) \geq \operatorname{dim} R-1$, then $\delta\left(R / \mathrm{m}^{2}\right)=1$ if and only if $R$ has minimal multiplicity.
\end{abstract}

\section{INTRODUCTION}

Throughout this paper we assume that $(R, \mathfrak{m}, k)$ is a commutative Noetherian Gorenstein local ring (or a graded Gorenstein $k$-algebra with unique maximal graded ideal $\mathfrak{m}$ ), and all modules are finitely generated. For an $R$-module $M$, there is an exact sequence of $R$-modules

$$
0 \longrightarrow Y_{M} \longrightarrow X_{M} \stackrel{\varphi}{\longrightarrow} M \longrightarrow 0
$$

where $X_{M}$ is a maximal Cohen-Macaulay module, $Y_{M}$ is a module of finite projective dimension, and $\varphi$ is right minimal, i.e., all endomorphisms $\alpha: X_{M} \rightarrow X_{M}$ with $\varphi \circ \alpha=\varphi$ are isomorphisms (see [1,2]). This sequence is called the minimal Cohen-Macaulay approximation of $M$. It is uniquely determined (up to isomorphism) by $M$. The theory of Cohen-Macaulay approximations was initiated by Auslander and Buchweitz. The rank of a maximal free direct summand of $X_{M}$ is denoted by $\delta_{R}(M)$ (or simply $\delta(M)$ ) and is called the $\delta$-invariant of $M$ (over $R$ ) by Auslander.

One of the main questions is how the $\delta$-invariant of $M$ reflects the structures of $M$ and of $R$. If $M$ is a module of finite projective dimension, the minimal Cohen-Macaulay approximation of $M$ is just the minimal free resolution of $M$. If an $R$-module $M$ has a factor module which is of finite projective dimension, then $\delta(M)>0[1,3]$. Thus the $\delta$-invariant of $M$ can be regarded as a measure of how far the module $M$ is from being of finite projective dimension. It was first conjectured that for an $R$-module $M, \delta(M)>0$ if and only if $M$ has a factor module of finite projective dimension. Unfortunately this is not the

Received by the editors July 22, 1992.

1991 Mathematics Subject Classification. Primary 13H10, 13C14, 13 A15. 
case in general (see the example in $\S 2$ ). However, the modules of the form $M=R / \mathfrak{m}^{n}, n \geq 1$, seem special. Following [3] and [5] we showed in [4] that if the associated graded ring $G(R)$ of $R$ is Cohen-Macaulay then $\delta\left(R / \mathrm{m}^{n}\right)=1$ if and only if $\mathrm{m}^{n}$ is contained in a parameter ideal of $R$. Here an ideal $I$ is called a parameter ideal if it is generated by a system of parameters of $R$. We then conjectured that this is true in general, i.e., without the assumption on $G(R)$. In this paper we give further evidence for the conjecture. We show that if $R$ is a graded Gorenstein $k$-algebra such that $\operatorname{depth} G(R) \geq \operatorname{dim} R-1$, then the conjecture holds for $R$. We call a local ring $(R, \mathfrak{m}, k)$ gradable if the completion $\hat{R}$ of $R$ at its maximal ideal $\mathfrak{m}$ is is omorphic to the completion of a graded $k$-algebra at the graded maximal ideal. Since the $\delta$-invariants behave well under faithfully flat ring extensions [1], we have that the conjecture holds for a Gorenstein local ring $R$ if $R$ is gradable and depth $G(R) \geq \operatorname{dim} R-1$.

It is known that $\delta(R / \mathfrak{m})=1$ if and only if $R$ is a regular local ring [3]. We show that for a Gorenstein local ring $R$ with $\operatorname{depth} G(R) \geq \operatorname{dim} R-1$, $\delta\left(R / \mathfrak{m}^{2}\right)=1$ if and only if $\mathfrak{m}^{2}$ is contained in a parameter ideal of $R$. If $R$ is not regular, then by a result of Sally [6], $R$ has minimal multiplicity.

The results in this paper are based on a particular description of the minimal Cohen-Macaulay approximation of a module $M$ with $\operatorname{depth} M=\operatorname{dim} R-1$. We give the description in $\S 1$. If $\operatorname{depth} G(R)=\operatorname{dim} R-1$, then by a result of [4], we can always reduce to the case where the dimension of $R$ is 1 . We thus devote $\S 2$ to giving various properties and examples of the $\delta$-invariants of cyclic modules over a 1-dimensional Gorenstein local ring. Using the properties we develop in $\S 2$, we prove the main results in $\S 3$.

\section{A CONSTRUCtion of Minimal COHEN-MaCAUlay approximation}

In this section we assume that $(R, \mathfrak{m}, k)$ is a Gorenstein local ring. We denote by $\mu(M)$ the minimal number of generators of an $R$-module $M$. Let $M$ be an $R$-module with depth $M=\operatorname{dim} R-1$. The following description of the minimal Cohen-Macaulay approximation of $M$ seems well known.

Proposition 1.1 [1]. Let $M$ be an $R$-module with $\operatorname{depth} M=\operatorname{dim} R-1$, and let $0 \rightarrow Y_{M} \rightarrow X_{M} \rightarrow M \rightarrow 0$ be the minimal Cohen-Macaulay approximation of $M$. Then $Y_{M} \cong R^{n}$, where $n=\mu\left(\operatorname{Ext}^{1}(M, R)\right)$.

Proof. Since $M$ is not a maximal Cohen-Macaulay module, we have

$$
\operatorname{Ext}^{1}(M, R) \neq(0) \text {. }
$$

Let $\xi_{1}, \ldots, \xi_{n}$ be a minimal set of generators of $\operatorname{Ext}^{1}(M, R)$, and let $E$ be the extension of $M$ by $R^{n}$ corresponding to the element $\left(\xi_{1}, \ldots, \xi_{n}\right)$ of $\operatorname{Ext}^{1}\left(M, R^{n}\right) \cong \bigoplus_{n} \operatorname{Ext}^{1}(M, R)$. Applying the functor $\operatorname{Hom}(, R)$ to the exact sequence

$$
0 \longrightarrow R^{n} \longrightarrow E \longrightarrow M \longrightarrow 0
$$

yields a long exact sequence

$$
\begin{aligned}
& 0 \rightarrow \operatorname{Hom}(M, R) \rightarrow \operatorname{Hom}(E, R) \rightarrow \operatorname{Hom}\left(R^{n}, R\right) \\
& \stackrel{\psi}{\longrightarrow} \operatorname{Ext}^{1}(M, R) \rightarrow \operatorname{Ext}^{1}(E, R) \rightarrow 0,
\end{aligned}
$$


and $\operatorname{Ext}^{i}(E, R)=(0)$ for $i \geq 2$. By the choice of $E$, the map $\psi$ is surjective. Therefore, $\operatorname{Ext}^{1}(E, R)=(0)$. Hence $E$ is a maximal Cohen-Macaulay $R$ module. Since $n=\mu\left(\operatorname{Ext}^{1}(M, R)\right),(1)$ in fact is the minimal Cohen-Macaulay approximation of $M$.

As an application of Proposition 1.1, we have the following result on the $\delta$-invariant of $M$.

Proposition 1.2. Let $M$ be an $R$-module with $\operatorname{depth} M=\operatorname{dim} R-1$. Then

$$
\delta(M)=\mu(M)+\mu\left(\operatorname{Ext}^{1}(M, R)\right)-\mu(\operatorname{Hom}(\Omega(M), R))
$$

where $\Omega(M)$ is the first syzygy of $M$.

Proof. We put $s=\mu(M), n=\mu\left(\operatorname{Ext}^{1}(M, R)\right)$, and $t=\mu(\operatorname{Hom}(\Omega(M), R)$. We have the short exact sequence $0 \rightarrow \Omega(M) \rightarrow R^{s} \rightarrow M \rightarrow 0$. Let $0 \rightarrow R^{n} \rightarrow$ $X_{M} \rightarrow M \rightarrow 0$ be the minimal Cohen-Macaulay approximation of $M$. We can construct the following pullback diagram:

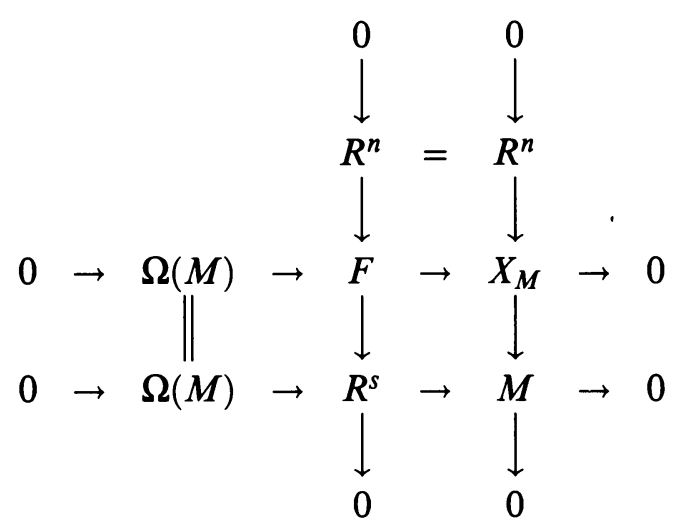

Thus we have $F \cong R^{n} \oplus R^{s}$. On the other hand, $\Omega(M)$ is a maximal CohenMacaulay $R$-module. Applying the functor $\operatorname{Hom}(, R)$ to the middle row, we obtain an exact sequence

$$
0 \longrightarrow \operatorname{Hom}\left(X_{M}, R\right) \longrightarrow \operatorname{Hom}(F, R) \longrightarrow \operatorname{Hom}(\Omega(M), R) \longrightarrow 0 .
$$

Therefore, $F \cong R^{t} \oplus R^{\delta(M)}$. Hence, we get $\delta(M)+t=s+n$.

\section{ONE-DIMENSIONAL RINGS}

In this section $(R, \mathfrak{m}, k)$ will always be a 1-dimensional Gorenstein local ring. For any $R$-module $M$, we denote by $\operatorname{soc}(M)$ the socle of $M$, i.e., $\operatorname{soc}(M)=\{x \in M \mid \mathfrak{m} x=0\}$. If $M$ is of finite length, we denote by $l(M)$ the length of $M$. We put $M^{*}=\operatorname{Hom}(M, R)$. In this section we always assume that $I$ is a regular ideal of $R$, i.e., the ideal $I$ contains a regular element of $R$. In this section we illustrate and apply the results in $\S 1$ to the cyclic modules over $R$.

First we have

Proposition 2.1. Let $I$ be an ideal of $R$. Then

$$
\delta(R / I)=1+l(\operatorname{soc}(R / I))-\mu\left(I^{*}\right) .
$$

In particular, we have $\delta(R / I)>0$ if and only if $l(\operatorname{soc}(R / I))=\mu\left(I^{*}\right)$. 
Proof. By Proposition 1.2 it suffices to show that $l(\operatorname{soc}(M))=\mu\left(\operatorname{Ext}^{1}(M, R)\right)$ for an $R$-module $\boldsymbol{M}$. Let $N$ be the maximal finite length submodule of $M$. Applying the functor $\operatorname{Hom}(, R)$ to the exact sequence $0 \rightarrow N \rightarrow M \rightarrow M / N \rightarrow$ 0 , we obtain an isomorphism $\operatorname{Ext}^{1}(N, R) \cong \operatorname{Ext}^{1}(M, R)$ since $M / N$ is a maximal Cohen-Macaulay module. Therefore, $l(\operatorname{soc}(M))=\mu\left(\operatorname{Ext}^{1}(N, R)\right)=$ $\mu\left(\operatorname{Ext}^{1}(M, R)\right)$. Since $\delta(R / I) \leq 1$ for any ideal $I$ of $R$ [1], we get the second statement.

Let $x \in \mathfrak{m}$ be an arbitrary $R$-regular element. The formula in Proposition 2.1 can be written in the following form.

Corollary 2.2. Let $x \in \mathfrak{m}$ be R-regular. Then

$$
\delta(R / I)=1+l(\operatorname{soc}(R / I))-l(\operatorname{soc}(I / x I)),
$$

and $\delta(R / I)=1$ if and only if $l(\operatorname{soc}(R / I))=l(\operatorname{soc}(I / x I))$.

Proof. We need only show that $\mu\left(I^{*}\right)=l(\operatorname{soc}(I / x I))$ for any regular element $x$ of $R$. The exact sequence $0 \rightarrow R \stackrel{x}{\rightarrow} R \rightarrow R / x R \rightarrow 0$ gives rise to an exact sequence $\left.0 \rightarrow I^{*} \stackrel{x}{\rightarrow} I^{*} \rightarrow \operatorname{Hom}_{R / x R}(I / x I, R / x R)\right) \rightarrow 0$. Since $x \in \mathfrak{m}$, we have $\mu\left(I^{*}\right)=\mu(\operatorname{Hom}(I / x I, R / x R))=l(\operatorname{soc}(I / x I))$.

As a consequence of the Proposition 2.1 we have

Corollary 2.3. Let $I$ be a Gorenstein ideal of $R$. Then $\delta(R / I)=1$ if and only if $I$ is a principal ideal.

Proof. Since $R / I$ has a simple socle, we have $\delta(R / I)=1$ if and only if $I^{*}$ is generated by one element. Hence $I^{*}$ is a free $R$-module of rank one. Applying the functor $\operatorname{Hom}(, R)$ to the exact sequence $0 \rightarrow I \rightarrow R \rightarrow R / I \rightarrow 0$ gives an exact sequence $0 \rightarrow R \rightarrow I^{*} \cong R \rightarrow \operatorname{Ext}^{1}(R / I, R) \rightarrow 0$. Therefore, $\operatorname{Ext}^{1}(R / I, R) \cong R /(x)$ for some regular element $x$ of $R$. Since $I$ is a Gorenstein ideal, we get $R / I \cong \operatorname{Ext}^{1}(R / I, R)$, and this implies that $I=(x)$.

Now we consider another case. Let $Q(R)$ be the total ring of quotients of $R$ with respect to the $R$-regular elements, and let $I^{-1}=\{z \in Q(R) \mid z I \subset R\}$. Then we know that there exists a natural $R$-isomorphism $\phi_{I}: I^{-1} \rightarrow I^{*}$ given by sending $z \in I^{-1}$ to the morphism in $I^{*}$ defined as multiplication by $z$. Also we have $I \subseteq I I^{-1} \subseteq R$. If $I I^{-1}=R$, then $I$ is a principal ideal and so $\delta(R / I)=1$. We now consider the case where $I I^{-1}=I$. We first give the following criterion.

Proposition 2.4. $\delta(R / I)=1$ if and only if $R \subset \mathrm{m} I^{-1}$.

Proof. We have the following commutative exact diagram

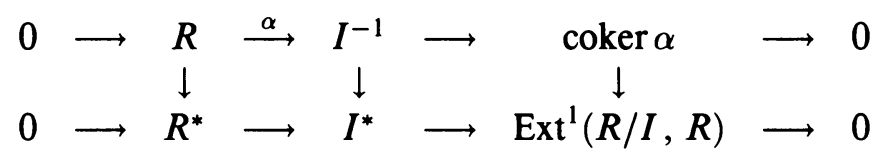

where $\alpha$ is the natural inclusion and all the vertical arrows are isomorphisms. By Proposition 2.1, $\delta(R / I)=1$ if and only if $\mu\left(I^{*}\right)=\mu\left(\operatorname{Ext}^{1}(R / I, R)\right)$. Hence we have $\delta(R / I)=1$ if and only if $\operatorname{Im} \alpha \subset \mathfrak{m} I^{-1}$.

As a consequence we have 
Corollary 2.5. If $I I^{-1}=I$, then $\delta(R / I)=0$.

Proof. We must show that $R \not \subset \mathrm{m} I^{-1}$. Suppose $R \subset \mathrm{m} I^{-1}$; then we have $I \subset \mathfrak{m} I^{-1} I \subset \mathfrak{m} I$ since $I I^{-1}=I$. This implies that $I=\mathfrak{m} I$, which is impossible by Nakayama's Lemma.

Remark. The condition $I I^{-1}=I$ in fact characterizes the conductors of the overrings of $R$ in $Q(R)$. Recall that for an overring $R^{\prime}$ with $R \subset R^{\prime} \subset Q(R)$, the conductor $\mathrm{c}$ of $R^{\prime}$ in $R$ is defined by $\mathrm{c}=\left\{z \in Q(R) \mid z R^{\prime} \subset R\right\}$. Here $\mathrm{c}$ is an ideal of $R$. It is easy to check that if $\mathrm{c}$ is the conductor of an overring $R^{\prime}$ in $R$, then $\mathrm{cc}^{-1}=\mathrm{c}$. Conversely, if $I I^{-1}=I$, then $I$ is the conductor of $\phi_{I}^{-1}(\operatorname{End}(I))$ in $R$, where $\phi_{I}: I^{-1} \rightarrow I^{*}$ is the natural isomorphism and $\operatorname{End}(I) \subset I^{*}$.

Now we return to the question in the introduction. If $I$ is contained in an ideal $J$ of finite projective dimension (in our situation $J$ has to be a principal ideal), then we know that $\delta(R / I)=1$. The converse is not true in general.

Example. Let $R=k\left[\left[t^{3}, t^{4}\right]\right]$ where $k$ is a field, and let $I=\left(t^{8}+t^{9}, t^{10}\right)$. It is not hard to check by direct calculation that

(a) $I$ is not contained in any principal ideal of $R$, and

(b) $l(\operatorname{soc}(R / I))=l\left(\operatorname{soc}\left(I / t^{3} I\right)\right)=2$.

Therefore, $\delta(R / I)=1+l(\operatorname{soc}(R / I))-l\left(\operatorname{soc}\left(I / t^{3} I\right)\right)=1$ by Corollary 2.2 , but $R / I$ has no factor module of finite projective dimension.

\section{THE CONJECTURE}

Let $(R, \mathfrak{m}, k)$ be a Gorenstein local ring (or a graded Gorenstein $k$-algebra with a unique graded maximal ideal $m$ ). We showed in [4] that if the associated graded ring $G(R)$ of $R$ is Cohen-Macaulay, then for any integer $n>0, \delta\left(R / \mathfrak{m}^{n}\right)=1$ if and only if there exists an $R$-sequence $\mathbf{x}$ such that $\mathfrak{m}^{n} \subset(\mathbf{x})$. Rings with $G(R)$ Cohen-Macaualy include hypersurface rings and homogeneous Gorenstein $k$-algebras. (A graded $k$-algebra is called homogeneous if it is generated by degree 1 elements over $k$.)

Conjecture. Let $(R, \mathfrak{m}, k)$ be a Gorenstein local ring (or a graded Gorenstein $k$-algebra with a unique graded maximal ideal $\mathfrak{m}$ ). For any integer $n>0$, $\delta\left(R / \mathfrak{m}^{n}\right)=1$ if and only if there is an $R$-sequence $\mathbf{x}$ such that $\mathfrak{m}^{n} \subset(\mathbf{x})$.

In this section we show two results related to the conjecture. In both cases we assume that $R$ satisfies the condition depth $G(R) \geq \operatorname{dim} R-1$. We first use the criterion for $\delta(R / I)=1$ that we developed in $\S 2$ to prove the one-dimensional case. Then we reduced the general case to the one-dimensional case by the result of [4].

Theorem 3.1. Let $R$ be a 1-dimensional graded Gorenstein $k$-algebra. For any graded ideal $I$ of $R, \delta(R / I)=1$ if and only if $I \subset(x)$, where $x$ is a homogeneous regular element of $R$. In particular, the conjecture holds for $R$.

Proof. According to Proposition 2.4, $\delta(R / I)>0$ if and only if $R \subset \mathrm{m} I^{-1}$. In particular, $\delta(R / I)>0$ implies that $1 \in \mathrm{m} I^{-1}$. Suppose $1=\sum_{i=1}^{l} z_{i} x_{i} / y_{i}$ where $x_{i}, y_{i}$, and $z_{i}$ are homogeneous elements of degree at least one and $x_{i} / y_{i} \in I^{-1}$ for all $i$. By comparing degrees we know that there is an $i$ such that 
$\operatorname{deg}\left(z_{i} x_{i} / y_{i}\right)=\operatorname{deg} z_{i}+\operatorname{deg} x_{i}-\operatorname{deg} y_{i}=0$. Therefore, we have $1=u z_{i} x_{i} / y_{i}$ with $u \in k$. This implies that $x_{i} / y_{i}=1 /\left(u z_{i}\right)$. Hence we get $I \subset\left(z_{i}\right)$.

Remark. Let $R$ be a 1-dimensional complete local Gorenstein domain containing an algebraically closed field $k$. It is known that $R$ is the completion of a graded $k$-algebra with respect to its irrelevant maximal ideal [7]. Therefore, the conjecture holds for such rings.

In [4] we showed the following, which allows us to use a reduction argument.

Lemma 3.2 [4]. Let $(R, \mathfrak{m}, k)$ be a Gorenstein local ring (or a graded Gorenstein $k$-algebra). Let $x \in \mathfrak{m} \backslash \mathfrak{m}^{2}$ be $R$-regular. Set $\bar{R}=R / x R$. Suppose the induced map $\bar{x}: \mathfrak{m}^{i-1} / \mathfrak{m}^{i} \rightarrow \mathfrak{m}^{i} / \mathfrak{m}^{i+1}$ is injective for $i \geq 0$. Then $\delta_{R}\left(R / \mathfrak{m}^{i}\right)=1$ if and only if $\delta_{\bar{R}}\left(R /\left(\mathfrak{m}^{i}, x\right)\right)=1$ for $i \geq 1$.

Combining this lemma and Theorem 3.1 we now obtain

Corollary 3.3. Let $(R, \mathfrak{m}, k)$ be a graded Gorenstein $k$-algebra. Let $G(R)$ be the associated graded ring of $R$. If $\operatorname{depth} G(R) \geq \operatorname{dim} R-1$, then the conjecture holds for $R$.

Recall that a local ring $(R, \mathfrak{m}, k)$ is said to be gradable if the completion $\widehat{R}$ of $R$ at its maximal ideal $\mathfrak{m}$ is isomorphic to the completion of a graded $k$-algebra at its unique maximal graded ideal. Since the $\delta$-invariants behave well under faithfully flat ring extensions [1], we have

Corollary 3.4. Let $(R, \mathfrak{m}, k)$ be a Gorenstein local ring. If $R$ is gradable and $\operatorname{depth} G(R) \geq \operatorname{dim} R-1$, then the conjecture holds for $R$.

Example. Let $R=k[[x, y, z]] / I$ where $I=\left(x^{3}+y^{9}, x^{2} z^{4}+y^{7}\right)$. Then $G(R)=k[x, y, z] / J$, with $J=\left(x^{3}, x^{2} z^{4}, x y^{7}, y^{14}\right)$, is not Cohen-Macaulay. However, it is easily checked that $R$ is gradable. This gives an example where $G(R)$ is not Cohen-Macaulay, but the conjecture holds for $R$. In this case $\delta\left(R / \mathfrak{m}^{i}\right)=0$ for $i \leq 7$ and $\delta\left(R / \mathfrak{m}^{8}\right)=1$.

It is known that $\delta(R / \mathfrak{m})=1$ implies that $\mathfrak{m}$ is contained in a parameter ideal of $R$. Therefore, $R$ is a regular local ring. In dealing with the case where $\delta\left(R / \mathfrak{m}^{2}\right)=1$, we have

Theorem 3.5. Let $(R, \mathfrak{m}, k)$ be a 1-dimensional Gorenstein local ring. Then $\delta\left(R / \mathfrak{m}^{2}\right)=1$ if and only if $\mathfrak{m}^{2} \subset(x)$ for some $x \in R$.

Proof. The "if" part is obvious. Now let $\delta\left(R / \mathfrak{m}^{2}\right)>0$. Set $I=\mathfrak{m}^{2}$. By Proposition 2.4 we have that $\delta(R / I)>0$ if and only if $R \subset \mathrm{m} I^{-1}$ if and only if $1=\sum r_{i} u_{i}$, where $r_{i} \in \mathfrak{m}$ and $u_{i} \in I^{-1}$. We define

$$
l=\min \left\{n \mid 1=\sum_{i=1}^{n} r_{i} u_{i}, r_{i} \in \mathfrak{m}, u_{i} \in I^{-1}\right\} .
$$

To show that $\mathfrak{m}^{2} \in(x)$ for some $R$-regular element $x$ is equivalent to showing that $l=1$. We now assume that $l>1$ and $\mu(\mathfrak{m}) \geq 3$ and derive a contradiction. (If $\mu(\mathfrak{m})=2$, then $R$ is a hypersurface and we know the conjecture is true.) 
Let $t \in \mathfrak{m} \backslash \mathfrak{m}^{2}$ be $R$-regular. Then $u_{i}$ can be written in the form $u_{i}=z_{i} / t^{2}$ with $z_{i} \in R$. We assume that all $z_{i}$ are in $\mathfrak{m}$ (otherwise, we have $l=1$ ). Thus we have

$$
1=\frac{\sum r_{i} z_{i}}{t^{2}}+\frac{\sum s_{i} x_{i}}{t}
$$

where $z_{i} / t^{2}, x_{i} / t$ are in $I^{-1}$. We assume that $r_{i}, s_{i}, z_{i}, x_{i} \in \mathfrak{m}$ (otherwise, $l=1)$ and $z_{i}, s_{i} x_{i} \notin t R$.

Now consider $z_{i} / t^{2} \in I^{-1}$ with $z_{i} \in \mathfrak{m}$ and $z_{i} \notin t R$. We have $z_{i} \mathfrak{m} / t=$ $z_{i} t \mathfrak{m} / t^{2} \subset R$, i.e., $z_{i} \mathfrak{m} \subset t R$. Therefore, under the reduction map $R \rightarrow \bar{R}=$ $R / t R$, we have $\overline{z_{i}} \in \operatorname{soc}(R / t R)$. Let $z_{j} / t^{2}$ be another element in $I^{-1}$ with $z_{j} \in \mathfrak{m}$ and $z_{j} \notin t R$. The same argument shows that $\overline{z_{j}} \in \operatorname{soc}(R / t R)$. Since $R / t R$ has simple socle, we get $z_{j}=a z_{i}+t y_{j}$ where $a \in R$ and $y_{j} \in R$. This implies that $y_{i} / t \in I^{-1}$. Hence (2) can be written in the form

$$
1=\frac{r z}{t^{2}}+\frac{\sum_{i=1}^{l-1} s_{i} x_{i}}{t}
$$

where $z / t^{2}, x_{i} / t$ are in $I^{-1}, r, s_{i} \in \mathfrak{m}$, and $z, s_{i} x_{i} \notin t R$. We assume that $z$ and $x_{i}$ are in $\mathfrak{m}$, and both $r z / t^{2}$ and $\sum s_{i} x_{i} / t$ are not in $\mathfrak{m}$ (otherwise, we are done).

Since $\sum s_{i} x_{i} \notin t R$, we may assume that $s_{1} x_{1} \notin t R$. However, we have $s_{1} x_{1} \mathfrak{m} \subset t R$, that is, under the reduction map $R \rightarrow R / t R, \overline{s_{1} x_{1}} \in \operatorname{soc}(R / t R)$. Since $R$ is Gorenstein, we get $s_{1} x_{1}=a z+t y$ with $a, y \in R$ and so

$$
\frac{s_{1} x_{1}}{t}=\frac{a t z}{t^{2}}+y \text {. }
$$

If $y \in \mathfrak{m}$, we get

$$
1-y=\frac{(r+a t) z}{t^{2}}+\frac{\sum_{i=2}^{l-1} s_{i} x_{1}}{t},
$$

contrary to the minimality of $l$. Therefore, $y$ is a unit in $R$ and (3) then can be written in the form

$$
1=\frac{r z}{t^{2}}+\frac{u x}{t}
$$

where $z / t^{2}, x / t$ are in $I^{-1}, z, x, r, u \in \mathfrak{m}$, and $z, x \notin t R$. We now consider the two induced linear maps

$$
\bar{x}: \mathfrak{m} / \mathfrak{m}^{2} \rightarrow R / t R \text { and } \bar{z}: \mathfrak{m} / \mathfrak{m}^{2} \rightarrow R / t^{2} R .
$$

We have that $\operatorname{Im} \bar{x} \subset \operatorname{soc}(R / t R)$ and $\operatorname{Im} \bar{z} \subset \operatorname{soc}\left(R / t^{2} R\right)$. Therefore,

$$
\operatorname{dim}_{k} \operatorname{Ker} \bar{x} \geq \mu(\mathfrak{m})-1 \text { and } \operatorname{dim}_{k} \operatorname{Ker} \bar{z} \geq \mu(\mathfrak{m})-1 .
$$

Since $\mu(\mathfrak{m}) \geq 3$, we have $\operatorname{Ker} \bar{x} \cap \operatorname{Ker} \bar{z} \neq(0)$. Thus there exists $s \in \mathfrak{m} \backslash \mathfrak{m}^{2}$ such that $z s \in t^{2} R$ and $x s \in t R$. Suppose $z s=t^{2} y, x s=t w$. Then $w \in \mathfrak{m}$. If $y$ is a unit of $R$, then $s$ is $R$-regular and $z / t^{2}=y / s$. This implies that $\mathfrak{m}^{2} \subset(s)$ and $l=1$. Suppose $y \in \mathfrak{m}$; then multiplying both sides of (5) by $s$ yields $s=r y+u w \in \mathfrak{m}^{2}$. This is a contradiction since $s \in \mathfrak{m} \backslash \mathfrak{m}^{2}$, and the proof is complete. 
Combining this result and Lemma 3.2 we obtain the following.

Corollary 3.6. Let $(R, \mathfrak{m}, k)$ be a Gorenstein local ring. Suppose that

$$
\operatorname{depth} G(R) \geq \operatorname{dim} R-1 \text {. }
$$

Then $\delta\left(R / \mathfrak{m}^{2}\right)=1$ if and only if $\mathfrak{m}^{2}$ is contained in a parameter ideal of $R$. In particular, if $R$ is not regular, then $\delta\left(R / \mathrm{m}^{2}\right)=1$ if and only if $R$ has minimal multiplicity.

\section{ACKNOWLEDGMENT}

I would like to thank Professor Maurice Auslander for introducing me to this subject and for stimulating discussions during the preparation of this paper.

\section{REFERENCES}

1. M. Auslander, Minimal Cohen-Macaulay approximations (in preparation).

2. M. Auslander and R. O. Buchweitz, The homological theory of maximal Cohen-Macaulay approximations, Mém. Soc. Math. France (N.S.), no. 38, Soc. Math. France, Paris, 1989, pp. 5-37.

3. S. Ding, A note on the index of Cohen-Macaulay local rings, Comm. Algebra 21 (1993), 53-71.

4. Math. Soc. (to appear)

5. J. Herzog, On the index of a homogeneous Gorenstein ring, preprint, 1992.

6. J. Sally, Tangent cones at Gorenstein singularities, Comput. Math. Appl., vol. 40, Academic Press, New York, 1980, pp. 169-175.

7. K. Watenabe, Some examples of one dimensional Gorenstein domains, Nagoya Math. J. 49 (1973), 101-109.

Department of Mathematics, Texas Tech University, Lubbock, TeXas 79409-1042

Current address: Department of Mathematics, Michigan State University, East Lansing, Michigan 48824

E-mail address, Michigan University: ding@math.msu.edu

E-mail address, Texas Tech University: sding@ttmath.ttu.edu 